\title{
Measurement-based solution for low frequency oscillation analysis
}

\author{
Chen SHEN ${ }^{1,2}$, Zhi AN ${ }^{1,2}$, Xianzhong DAI ${ }^{1,2}$, Wei WEI ${ }^{1,2,3}$, \\ Lijie DING ${ }^{3}$
}

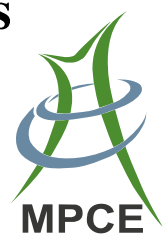

\begin{abstract}
This paper presents a measurement-based solution for low frequency oscillation (LFO) analysis in both real time monitoring and off-line case study. An online LFO property discrimination method is developed first, which alternately uses empirical mode decomposition (EMD)/Hilbert transform (HT) and square calculation to process the measurement data. The method magnifies the variation trend of oscillating variables to accurately discriminate the property of the oscillation. Subsequently, an oscillation source locating method for the forced oscillation (FO) and a strongly correlated generator identification method for the weak damping oscillation (WDO) are proposed. Finally, numerical study results on a test system of
\end{abstract}

CrossCheck date: 5 April 2016

Received: 29 September 2015/Accepted: 5 April 2016/Published online: 7 May 2016

(c) The Author(s) 2016. This article is published with open access at Springerlink.com

$\triangle$ Chen SHEN

shenchen@mail.tsinghua.edu.cn

Zhi AN

thuaz07@gmail.com

Xianzhong DAI

happydxz1@126.com

Wei WEI

weiwei_6898825@163.com

Lijie DING

ding_lijie@163.com

1 Department of Electrical Engineering, Tsinghua University, Beijing, China

2 State Key Laboratory of Control and Simulation of Power Systems and Generation Equipments, Tsinghua University, Beijing, China

3 Electric Power Research Institute of Sichuan Electric Power Company, Chengdu, China the isolated Changdu grid in Tibet validate the proposed methods.

Keywords Low frequency oscillation, Oscillation property discrimination, Oscillation source location, Strongly correlated generator identification

\section{Introduction}

Low frequency oscillation (LFO) is a common phenomenon in power systems. It has been a serious problem limiting the transmission power of tie-lines and threatening the security and stability of power systems [1-7]. It is crucial to discover the origin of LFO accurately and to generate corresponding control strategies effectively.

The LFOs are generally classified into two categories, the weak damping oscillations (WDO) and the forced oscillations (FO), according to different intrinsic inducements [811]. WDOs occur in systems with weak or negative damping so that the oscillation can hardly calm down. FOs are caused by oscillatory sources with approximate frequency to a system's inherent mode. These two kinds of oscillations have similar appearance. However, the corresponding control measures are entirely different. To restrain the WDO, the output power of some strongly correlated generators needs to be decreased temporarily if the oscillation mode is unexpected, and thereafter controllers such as power system stabilizers (PSS) need to be added or retuned; whereas to attenuate the FO, the oscillation source needs to be isolated or tripped. Therefore, it is essential to discriminate oscillation properties when an LFO is detected.

Eigen-analysis is a major approach in LFO study [12-14]. This approach heavily depends on the availability of power system models and parameters. However, with the expansion of system scales, to obtain parameters of all system devices 
becomes harder than before. Moreover, it is very difficult to ensure computational convergence and accuracy when calculating the modes and mode-shapes of a large system. Recent years, enormous progresses have been made in measurementbased oscillation analysis because of the rapid development of wide-area measurement system (WAMS). In identifying oscillation properties, the characteristics of waveforms in different oscillation period were analyzed in [8]. Reference [9] focused on the initial period of the waveforms, proposing a second order differential method. The oscillation properties were discriminated according to the components of power response in [10]. Reference [11] decomposed the energy supply on port (ESP) and discriminated the oscillation properties according to the features of ESP's aperiodic component. As for the oscillation source locating, the concept of generator energy was firstly introduced and successfully used to identify the generator where the external disturbance exists in [15]. Further, [16-18] proposed the concept of energy flow in the network, based on which the oscillation sources could be located correctly on a generator or a load bus. Different from the energy definition of the above references, [19] defined the total energy of power system based on Hamiltonian realization, and the external disturbance could be located effectively in large-scale power systems containing complicated control devices.

This paper proposes a method to discriminate oscillation properties. Combining this method with the previous work of the authors' research group [19-22], this paper forges a measurement-based solution for LFO analysis. There are three main steps in this solution. First, an online discriminating method is developed, which alternately uses the empirical mode decomposition (EMD) / Hilbert transform (HT) and square calculation to process the measurement data. With this method, an oscillation is promptly classified into WDO or FO. In the next step, the oscillation source is located for the FO, or subsystems that contribute more to the underdamped or negative-damping mode are identified for the WDO. The third step is, only for the WDO, to locate generators that are strongly correlated to the oscillation. This solution not only helps to generate prompt control strategies in real time operation, but also helps to identify the generators that should equip a PSS or retune the parameters of the PSS if they have already equipped one. In the final part of this paper, the solution is applied to an oscillation case found in simulation on the isolated Changdu grid in Tibet to demonstrate its effectiveness.

\section{Oscillation property discrimination method based on alternate use of EMD/HT and square calculation}

A measurement-based discrimination method based on alternate use of EMD/HT and square processing is proposed, which only requires online measurements such as transmission power on tie-lines or voltage angles at buses. It can discriminate the oscillation properties within a very short time and help generating online control strategies.

\subsection{Mathematical tools}

\subsubsection{Empirical mode decomposition}

The empirical mode decomposition can decompose sample data into $n$-intrinsic mode functions (IMFs) and a residue that could be either the mean trend or a constant [23]. The EMD has been widely used in power oscillations or signal analysis combining with the HT [24-28].

The EMD decomposes the sample into

$x(t)=\sum_{i=1}^{n} c_{i}(t)+r(t)$

where $x(t)$ is the sample data; $c_{i}(t)$ is $i^{\text {th }}$ intrinsic mode function; $r(t)$ is the residue.

If the sample includes several oscillation modes, IMF results are physically meaningful: the $i^{\text {th }}$ IMF is corresponding to the $i^{\text {th }}$ oscillation mode, i.e.,

$c_{i}(t)=A_{i} \mathrm{e}^{\sigma_{i} t} \sin \left(\omega_{i} t+\varphi_{i}\right)$

where $A_{i}$ is a constant; $\sigma_{i}$ and $\omega_{i}$ are the real-part and the imaginary part of the $i^{\text {th }}$ mode; $\varphi_{i}$ is the initial phase.

In this section, the EMD is repeatedly used to get the oscillation component of data.

\subsubsection{Hilbert transform}

The HT of $x(t)$ is defined in [23], that is

$\hat{x}(t)=\frac{1}{\pi} x \int_{-\infty}^{+\infty} \frac{x(\tau)}{t-\tau} \mathrm{d} \tau$

With the HT, the analytic signal is defined as

$z(t)=x(t)+\mathrm{j} \hat{x}(t)=A(t) \mathrm{e}^{i \theta(t)}$

The instantaneous amplitude of $x(t)$ is calculated by

$A(t)=\left(x^{2}(t)+\hat{x}^{2}(t)\right)^{\frac{1}{2}}$

The HT is used to get the instantaneous amplitude of the oscillation components of the data.

\subsection{Theoretic foundation of method for oscillation property discrimination}

In a practice power system comprising of several interconnected areas, it usually has only one key mode whose damping is the weakest, especially when the connections between areas are weak while the connections within areas are very strong. After the attenuation of modes with large 
damping ratios, only the weak damping mode, called the dominant mode, or the forced response remains.

For free oscillation, a state variable is as follows.

$x_{\mathrm{d} \_\mathrm{WDO}}(t)=A \mathrm{e}^{\sigma t} \sin (\omega t+\varphi)$

For forced oscillation, a state variable is

$x_{\mathrm{d} \_ \text {FO }}(t)=A \sin (\omega t+\varphi)$

The amplitude of (6) changes exponentially with the certain directional trend. The amplitude of (7) keeps constant in ideal condition or fluctuating around a constant value due to some noises. Anyway, it varies without any directional trend.

According to (6) and (7), whether the amplitude varies with a certain directional trend could be used as the key discriminative characteristic to identify the oscillation properties. The problem is that the discriminative characteristic is not so prominent in the raw data obtained from the two kinds of oscillations. Additional work has to be done to enlarge the difference.

In this paper, a new method is proposed to process the raw data, which magnifies the difference by alternately using EMD and square calculation. The raw data are decomposed into $n$-IMFs and a residue. Then the instantaneous amplitude of every IMF is extracted by HT. Choose the IMF whose instantaneous amplitude is the biggest as the dominant mode and then normalize it by its instantaneous amplitude at $t=0$.

As for the weak damping oscillation, square $x_{\mathrm{d}}$ WDO $(t)$ and multiply the result by 2 ,

$2\left(x_{\mathrm{d} \_ \text {WDO }}(t)\right)^{2}=A^{2} \mathrm{e}^{2 \sigma t}(1-\cos (2 \omega t+2 \varphi))$

Abstract oscillatory component from (8) by EMD, then

$x_{\mathrm{d} \_\mathrm{WDO}}^{(1)}(t)=-A^{2} \mathrm{e}^{2 \sigma t} \cos (2 \omega t+2 \varphi)$

We define the processing by (8) and (9) as a round of operation. $x_{\mathrm{d}-\mathrm{WDO}}^{(k)}(t)$ is the result after $k$ times of operation.

After $n$ times of operation,

$x_{\mathrm{d} \_\mathrm{WDO}}^{(n)}(t)=(-1)^{n} A^{2^{n}} \mathrm{e}^{2^{n} \sigma t} \cos \left(2^{n} \omega t+2^{n} \varphi\right)$

The instantaneous amplitude of $x_{\mathrm{d} \text { WDO }}^{(k)}(t)$ is

$\operatorname{Amp}(t)=A^{2^{n}} e^{2^{n} \sigma t}$

It can be seen from (10) and (11) that the damping factor and oscillation frequency have been magnified by $2^{n}$. The variation trend of the amplitude has also been amplified and become steeper.

As for the forced oscillation, repeating square calculation and EMD alternately for $n$ times, finally we obtain

$x_{\mathrm{d} \_\mathrm{FO}}^{(n)}(t)=(-1)^{n} A^{2^{n}} \cos \left(2^{n} \omega t+2^{n} \varphi\right)$

The instantaneous amplitude of oscillation data processed by HT in (12) is
$\operatorname{Amp}(t)=A^{2^{n}}$

The oscillation frequency has also been amplified by $2^{n}$, however, the instantaneous amplitude remains constant.

The above analysis results are obtained in the ideal conditions. Factually, the practical oscillation data contain disturbances or noises, but thankfully they are generally irregular and nondirective, therefore they will not impact the variation trend of the instantaneous amplitude. So in the practical conditions, (11) and (13) might have the general forms as (14) and (15), respectively.

$A m p(t)=a \mathrm{e}^{b t}+c$

$A m p(t)=a t+b$

where $a, b, c$ are constants.

After the above process, the discriminative characteristic between the two oscillation properties has become significant enough for discrimination.

\subsection{Implementation process of method}

Generally, the sample data contain noises, therefore they should be filtered at first. Then if there is a dominate mode, it should be separated from others by EMD. Besides, if a system has multiple modes excited simultaneously, the dominate mode should be separated from others by the EMD. The systematic implementation process of the method proposed above is as following.

1) Choose generators' active power as input data for oscillation type identification. A low-pass filter is used to filter the input data in order to eliminate the noises and the components whose frequency are beyond the range of electromechanical oscillations.

2) Get the dominant oscillation mode. Repeat square calculation and EMD alternately $n$ times.

3) Extract the instantaneous amplitude $A m p(t)$ by HT and fit it with (14) and (15) respectively. Then, the exponential fitting result $A m p_{\mathrm{e}}(t)$, the approximatively linear fitting result $A m p_{1}(t)$ and the goodness-of-fit statistics such as the sum of squares due to error (SSE), the root mean squared error (RMSE) and the coefficient of determination (CD) are obtained. Calculate the correlation coefficient $R$ between the instantaneous amplitude and the fitting data $A m p_{\mathrm{e}}(t)$ and $A m p_{1}(t)$ respectively.

4) Discriminate the oscillation properties with the goodness of fit and the correlation coefficients. If SSE and RMSE for exponential fitting are smaller than that for linear fitting and meanwhile $\mathrm{CD}$ and $R$ for exponential are larger, then the exponential fitting is more similar to instantaneous amplitude and we can conclude that the oscillation is a weak damping oscillation. 
Otherwise, if SSE and RMSE for linear fitting are smaller than that for exponential fitting and meanwhile $\mathrm{CD}$ and $R$ are larger, the property could be determined as an FO.

\section{Measurement-based solution for LFOs analysis in online monitoring and offline research}

\subsection{Oscillation source locating method in FOs}

The foremost affair to restrain LFOs or eliminate their hazards is to discover their properties. After discriminating the properties, as for the WDO, the next step is to find which component contributes most to the negative damping; for the FO, it is important to locate the oscillation source.

The authors' research group (2012) proposed an oscillation source locating method that could directly locate the oscillation source in the governors or exciters of generators when there is an FO [19-21] (This method can also be used to distinguish the governors or exciters that participate in a local mode with negative damping).

References [19-21] introduced the concept of ESP. It is a kind of transient energy derived from the port-controlled Hamiltonian theory, which is injected into the network at corresponding ports. By calculating ESP, the external disturbances could be distinguished as the oscillation source for a FO, or the subsystems that contribute more to the negative damping could be found out for a WDO.

\subsection{Strongly correlated generators identification method in WDOs}

When an unexpected weak damping oscillation occurs, which hasn't been detected in offline study for whatever reasons, the most efficient measure to restrain it is to reduce the output power of strongly correlated generators temporarily. Conventional eigen-analysis could provide information about mode shapes and participation factors (PF). Nevertheless, the oscillation modes in practical grids usually mismatch those in the simulations due to the unmodeled system dynamics or incorrect parameters. Therefore, an online method to identify strongly correlated generators is in urgent need for real-time control strategies generating when a WDO occurs.

The authors' research group (2015) proposed an online identification method based on the power supply on the port (PSP) [22], which is defined as the rate of change of ESP. A strong correlation index to assess how deep a generator participates in a WDO is defined as
$R_{\omega O i}(t)=A_{R_{\omega O i}}(t) \sin \left(2 \omega t+2 \varphi_{\delta_{i}}+\varphi_{B i}\right)$

where $R_{\omega O}(t)$ is the $i^{\text {th }}$ generator's periodic component of the PSP in the governor channel; $A_{R_{\omega O} i}(t)$ is the instantaneous amplitude of $R_{\omega O i}(t) ; \omega$ is the frequency; $\varphi_{\delta_{i}}$ is the initial phase of the oscillation; $\varphi_{B i}$ is the term occurred in trigonometric calculation. The $A_{R_{\omega O O}}(t)$ is defined as the strong correlation index, which could be extracted by HT.

Reference [22] also demonstrated that the index has the same distribution with the PF, so that it can be utilized as a standard to determine which generators' output should be reduced.

\subsection{Systemic implementation of solution}

With the methods respectively introduced in Sections 2, 3.1 and 3.2, a measurement-based systematic solution for the LFO analysis in online monitoring or offline research has been forged.

When an LFO is detected, the first step should be the oscillation property discrimination with the method based on the alternate EMD/HT and square processing. Next, the oscillation source should be located utilizing the method in Section 3.1 if the oscillation is regarded as an FO. If the oscillation is judged to be a WDO, the strongly correlated generators identification method should be utilized to find out the generators whose output should be reduced. The implementation process is shown in Fig. 1.

This solution is appropriate to both simulation researches and online monitoring for practical systems. As for the practical systems, the solution provides abilities to rapidly discover and locate the causal factors of an LFO, thus the

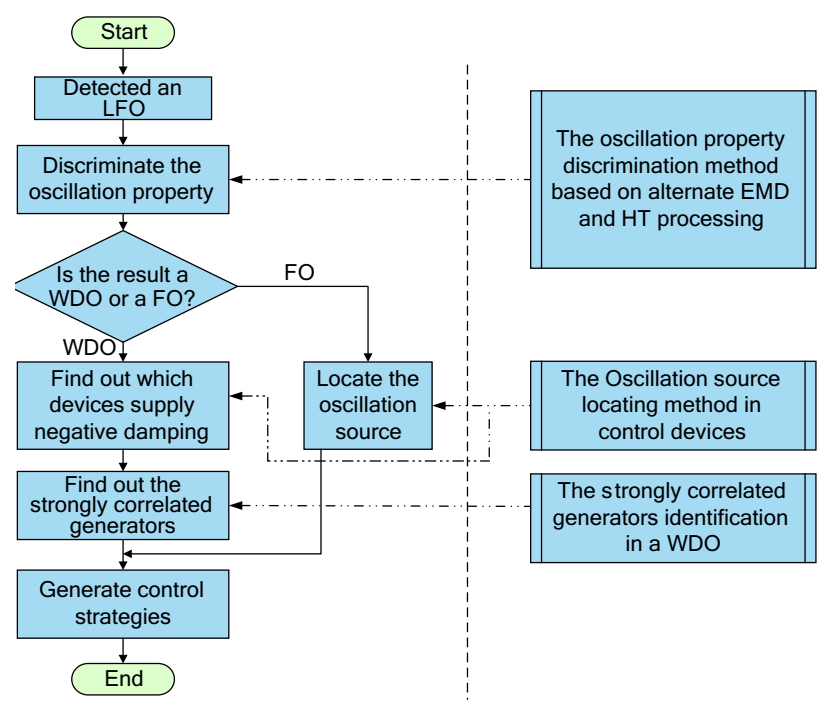

Fig. 1 Flow chart of measurement-based solution of LFO analysis 


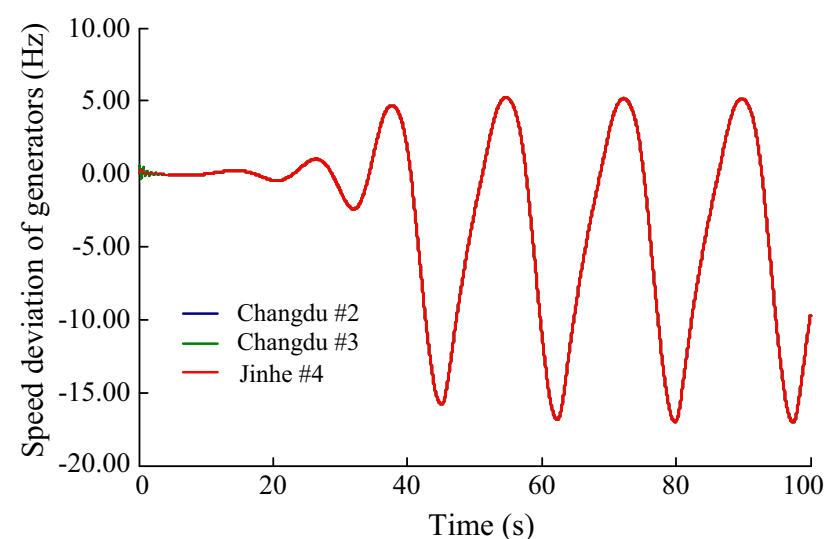

Fig. 2 Speed deviation of generators

control measures could be taken as soon as possible. While in offline researches like power system planning, it helps to find the devices with inappropriate models or parameters, so that the system dynamics could be improved.

\section{Case study}

\subsection{General information of Changdu case}

In the simulation study on the Sichuan-Tibet interconnection project, an uncommon phenomenon was found in the isolated grid of Changdu in Tibet, where there is a divergent frequency oscillation in the whole grid.

Changdu is located in the east of Tibet near Ganzi autonomous prefecture in Sichuan province. The grid has been operating in a standalone mode for years. The target of the interconnection project is to connect this isolated grid to the Sichuan grid to solve the power shortage in this area radically. In the case where there is a frequency oscillation, the isolated Changdu grid has 8 generators

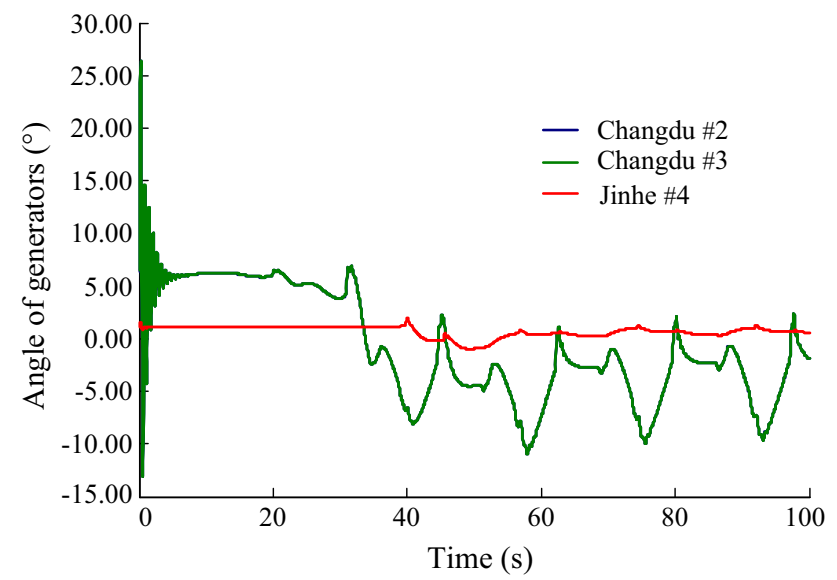

Fig. 3 Angle of generators (reference is Jinhe 2)

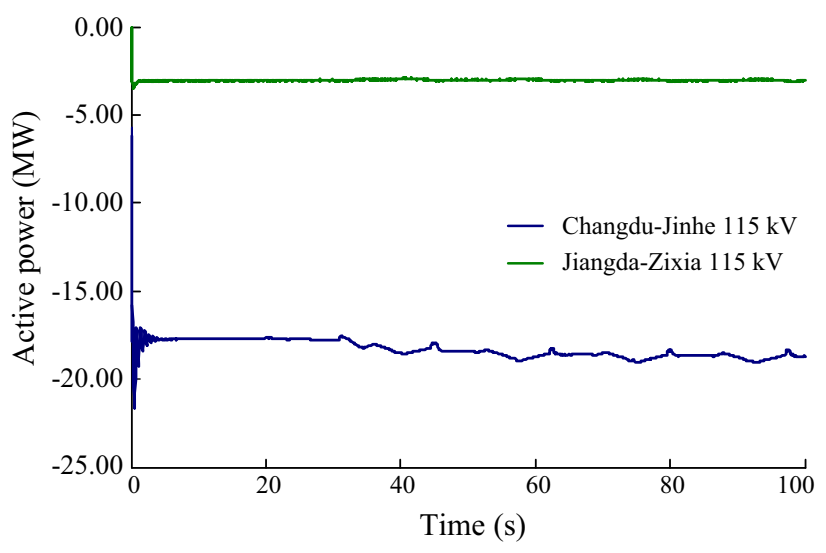

Fig. 4 Active power of tie-lines

located in 2 plants and each plant has 4 generators respectively, with the total output of $46 \mathrm{MW}$. Simulation results on a 3-phase-to-ground short circuit fault are shown in Fig. 2 to Fig. 4 (some curves overlap with each other). The fault lasts 5 circles and disappears thereafter.

These figures indicate that the generators do not lose synchronization but the frequency of the system has a swing with peak-to-peak value up to $20 \mathrm{~Hz}$, which means that the system cannot operate. Nonetheless, the actual system in Changdu has been operating in such a situation over years. So, there must be some inaccurate models or parameters in this simulation case and they must be found.

\subsection{Locating problem utilizing proposed solution}

\subsubsection{Oscillation property discrimination}

Noticing that the transmission power has less obvious fluctuation, the angles of generators should be chosen as the study object considering the efficiency of the algorithm.

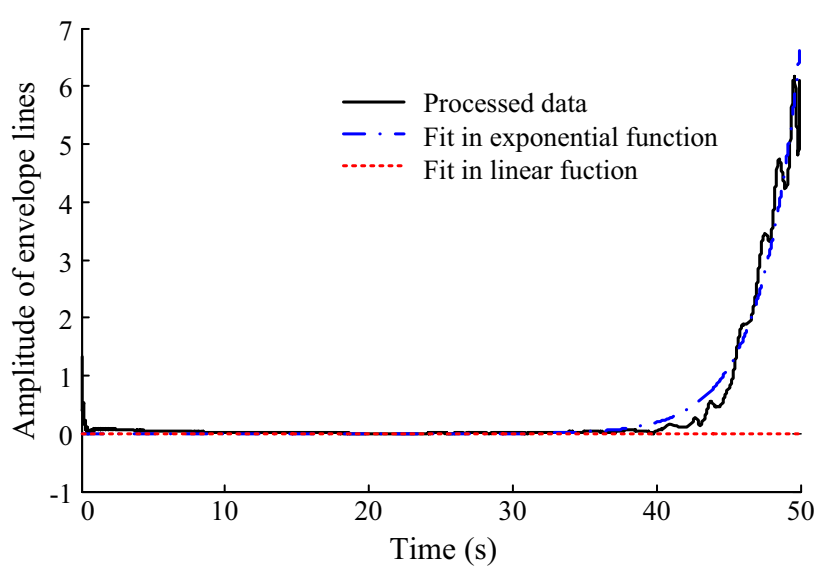

Fig. 5 Angle of generators (reference is Jinhe \#2) 
Table 1 Goodness of fit

\begin{tabular}{lllr}
\hline Expression & SSE & RMSE & CD \\
\hline$A(t)=a \mathrm{e}^{b t}+c$ & $1.6537 \times 10^{16}$ & $1.8194 \times 10^{6}$ & 0.9708 \\
$A(t)=a t+b$ & $7.5286 \times 10^{17}$ & $1.2025 \times 10^{7}$ & -0.1196 \\
\hline
\end{tabular}

The fit results are shown in Fig. 5, referring to (16) and (17) respectively. The results obviously imply that the fit in exponential function is better than in constant. The goodness of fit is shown in Table 1, which also verifies the conclusion.

It can be seen in Table 1 that the SSE and RMSE of the exponential fit are smaller than the linear fit, and meanwhile the $\mathrm{CD}$ and $R$ of the exponential fit are larger. Therefore, the oscillation is judged to be a WDO.

\subsection{Finding out negative damping}

Since the oscillation is a WDO, the next step is to find out which device contributes more to the negative damping.

Figure 6 shows that the governors in both plants has increasing and large ESP (generators in the same plant has almost the same response) in this case, where the Jinhe plant's ESP is larger. On the contrary, the exciters' ESP has much smaller magnitude. This result indicates that the governors are the devices that contribute more to the negative damping, and Jinhe plant might play a more important role in the oscillation.

\subsection{Identifying strongly correlated generators}

After the negative damping has been confirmed, the next problem is to determine which plant is the most critical one.

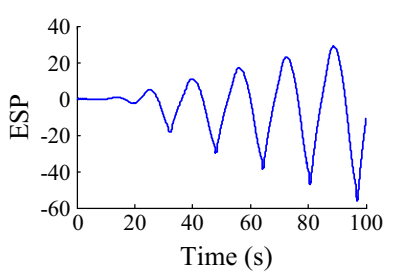

(a) Governor of Changdu \#2

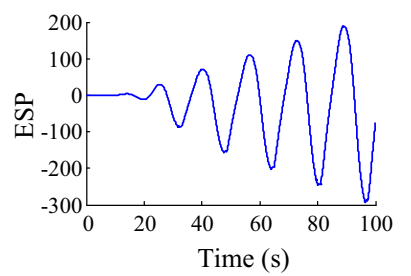

(c) Governor of Jinhe \#4

Fig. 6 ESP of some generators

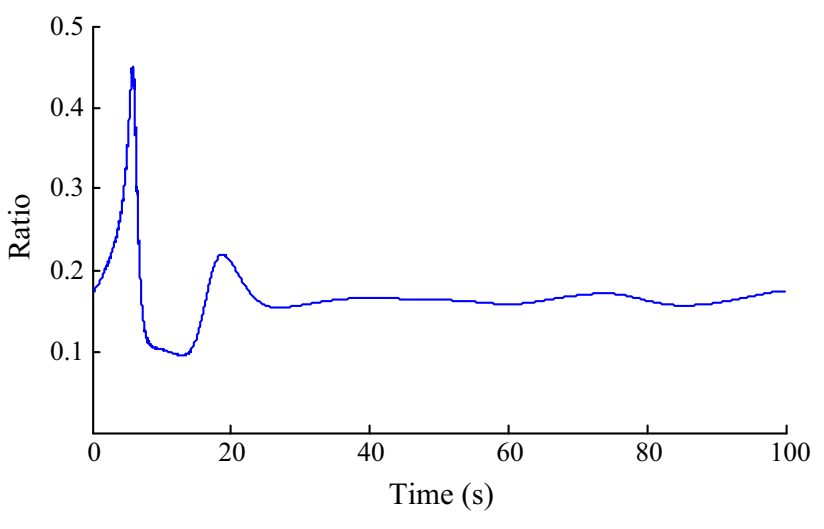

Fig. $7 A_{R_{\omega O i}}(t)$ ratio of Changdu \#2 to Jinhe \#4

Figure 7 shows that the $A_{R_{\omega O} i}(t)$ of Changdu is always less than that of Jinhe, which means that the Jinhe plant has stronger correlation to the oscillation.

\subsection{Comparison to traditional simulation}

After all the process, it can be concluded that the governors in Jinhe plant might be the cause for the negative damping.

In fact, the values of soft feedback constant $D_{\mathrm{d}}$ (in the numerator of soft feedback) of the governors in Jinhe is finally found to be inappropriate, so that the system forms a positive feedback, thus it becomes unstable. A Simulink model, which contains a synchronous generator and a detailed governor system with the same parameters as the original devices, is built to test the governor. The governor model (with the corresponding hydro-turbine) is shown in Fig. 8. This simulation is driven by a step change in rotating speed are shown in Fig. 9. It can be seen that with the original $D_{\mathrm{d}}$ the system is unstable, whereas the system is stable with a ten times larger $D_{\mathrm{d}}$.

(b) Exciter of Changdu \#2

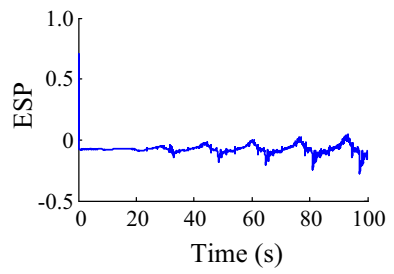

(d) Exciter of Jinhe \#4

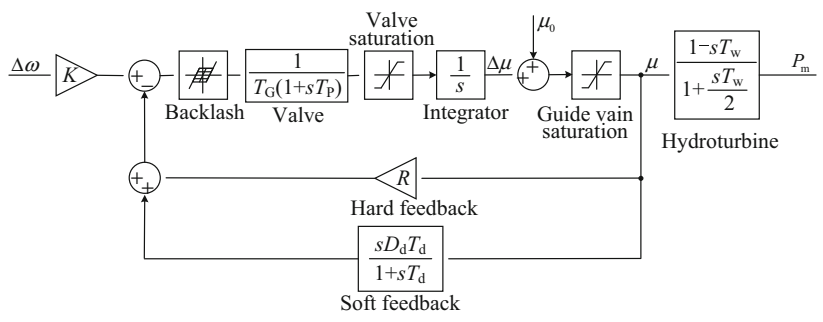

Fig. 8 Model of governor with hydro-turbine 


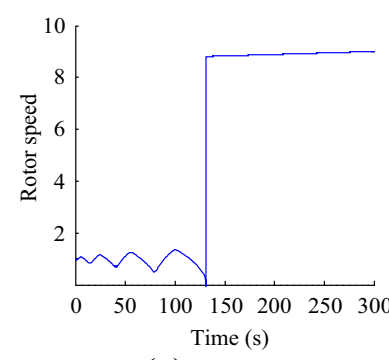

(a) $D_{\mathrm{d}}=0.0253$

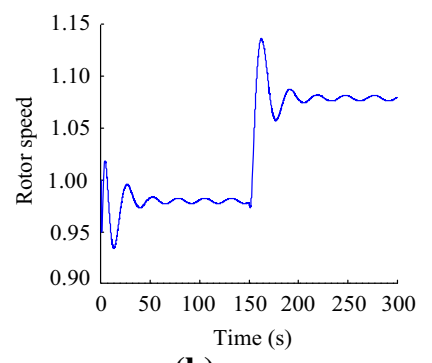

(b) $D_{\mathrm{d}}=0.253$
Fig. 9 Test results of different $D_{\mathrm{d}}$ utilized in governor

The results of eigen-analysis of the system with original $D_{\mathrm{d}}$ are shown in Table 2. There is a pair of eigen-values with positive real-part, which means the system is unstable. Meanwhile, the mode shapes information shown in Table 3 indicates that the modules of right eigenvector of all the generators are adjacent to 1 and the angles are adjacent to 0 , which means all the generators swing synchronously, complying with the phenomena observed and the conclusions obtained from the proposed measurement-based methods.

Table 2 Modes of isolated Changdu grid

\begin{tabular}{llc}
\hline Mode & Frequency $(\mathrm{Hz})$ & Damping ratio \\
\hline$-2.723 \pm \mathrm{j} 8.641$ & 1.375 & 0.301 \\
$-3.197 \pm \mathrm{j} 9.401$ & 1.496 & 0.322 \\
$-3.193 \pm \mathrm{j} 9.404$ & 1.497 & 0.322 \\
$-1.431 \pm \mathrm{j} 11.111$ & 1.768 & 0.128 \\
$-1.179 \pm \mathrm{j} 11.521$ & 1.834 & 0.102 \\
$-1.361 \pm \mathrm{j} 11.695$ & 1.861 & 0.116 \\
$-1.628 \pm \mathrm{j} 12.246$ & 1.949 & 0.132 \\
$\mathbf{0 . 1 4 0} \pm \mathbf{j 0 . 3 2 2}$ & 0.051 & -0.398
\end{tabular}

Table 3 Mode shape of unstable mode

\begin{tabular}{llr}
\hline Generator & Module of elements in right eigenvector & Angle \\
\hline Changdu \#0 & 1.0000 & 0.00 \\
Changdu \#2 & 1.0000 & 0.00 \\
Changdu \#3 & 1.0000 & 0.00 \\
Changdu \#5 & 1.0000 & 0.00 \\
Jinhe \#2 & 0.9892 & -0.12 \\
Jinhe \#4 & 0.9905 & -0.12 \\
Jinhe \#6 & 0.9904 & -0.12 \\
Jinhe \#8 & 0.9905 & -0.12 \\
\hline
\end{tabular}

\section{Conclusion}

In this article, an oscillation property discrimination method based on alternate EMD and HT is introduced, and a measurement-based solution for the LFO online monitoring and offline research is proposed. The solution includes a series of concrete methods from the oscillation property discrimination to the oscillation source location and the strongly correlated generator identification. With this solution, the causal factor of low frequency oscillation could be discovered and located rapidly. It is a compatible assistant for control strategies generating to restrain the oscillation or improve inappropriate models and parameters in simulation researches. The case study on Changdu grid proves the effectiveness of the solution.

Considering that multi oscillation modes might be excited after some certain faults and sometimes they could be too similar to be distinguished, this solution could be further refined if there is a methodology to effectively separate them, so that targeting control strategies could be obtained respectively.

Acknowledgment This work was supported in part by the National Natural Science Foundation of China (No. 51177079, No. 51321005) and Sichuan Electric Power Company.

Open Access This article is distributed under the terms of the Creative Commons Attribution 4.0 International License (http:// creativecommons.org/licenses/by/4.0/), which permits unrestricted use, distribution, and reproduction in any medium, provided you give appropriate credit to the original author(s) and the source, provide a link to the Creative Commons license, and indicate if changes were made.

\section{References}

[1] Schleif RF, White HJ (1966) Damping for the NorthwestSouthwest tie line oscillations: an analog study. IEEE Trans Power Appar Syst 85(12):1239-1247

[2] Bauer DL, Buhr WD, Cogswell SS et al (1975) Simulation of low frequency undamped oscillations in large power systems. IEEE Trans Power Appar Syst 94(2):207-213

[3] Rogers G (2000) Power system oscillations. Kluwer Academic Publishers, Boston

[4] Luo GJ, Xu XH, Long SQ et al (1986) Low frequency oscillations on the interconnectors between Hong Kong and Guangdong. PCSEE 6(1):29-35

[5] Wang MY (2008) The accident analysis on large scale power grid and the techniques application. China Electric Power Press, Beijing

[6] Liu GP, Xu Z, Huang Y et al (2004) Analysis of inter-area oscillations in the South China interconnected power system. Electr Power Syst Res 70(1):38-45

[7] Sun JQ, Chen ZG, Cao HZ (2007) Analysis on low-frequency oscillation of South China power grid in 2010. Power Syst Technol 31(S2):93-97

[8] Yang DJ, Ding JY, Shao HQ et al (2013) WAMS based characteristic discrimination of negative damping low-frequency 
oscillation and forced power oscillation. Autom Electr Power Syst 37(13):57-62. doi:10.7500/AEPS201208028

[9] Li Y, Jia WS, Li WF et al (2013) Online identification of power oscillation properties based on the initial period of wave. PCSEE 33(25):54-60

[10] Ye H, Song YB, Liu YT (2013) Forced power oscillation response analysis and oscillation type discrimination. PCSEE 33(34):197-204

[11] Dai XZ, Shen C (2014) A power system oscillation property identifying method based on decomposition of energy supply on port. Autom Electr Power Syst 38(23):40-45. doi:10.7500/ AEPS20140606007

[12] Kundur P (1994) Power system stability and control. McGrawHill, New York

[13] Anderson PM, Fauld AA (2012) Power system control and stability, 2nd edn. Wiley-IEEE Press, Hoboken

[14] Souza Lima EE, De Jesus Fernandes LF (2000) Assessing eigenvalue sensitivities. IEEE Trans Power Syst 15(1):299-306

[15] Yu YP, Min Y, Chen L et al (2010) Disturbance source location of forced power oscillation using energy functions. Autom Electr Power Syst 34(5):1-6

[16] Chen L, Min Y, Hu W (2012) Low frequency oscillation analysis and oscillation source location based on oscillation energy, Part one: mathematical foundation and energy flow computation. Autom Electr Power Syst 36(3):22-27

[17] Chen L, Chen YP, Min Y et al (2012) Low frequency oscillation analysis and oscillation source location based on oscillation energy, Part two: method for oscillation source location and case studies. Autom Electr Power Syst 36(4):1-5

[18] Chen L, Min Y, Hu W (2012) An energy-based method for location of power system oscillation source. IEEE Trans Power Syst 28(2):828-836

[19] Li Y, Shen C, Liu F (2012) An energy-based methodology for locating the source of forced oscillations in power systems. In: Proceedings of the 2012 IEEE international conference on power system technology (POWERCON'12), Auckland, 30 Oct2 Nov 2012, 6 pp

[20] Li Y, Shen C, Liu F (2012) Oscillation on source location in control devices of generators based on Hamilton realization. Autom Electr Power Syst 36(23): 6-11, 86

[21] Li Y, Shen C, Liu F (2013) A methodology for power system oscillation analysis based on energy structure. Autom Electr Power Syst 37(13):49-56. doi:10.7500/AEPS201211185

[22] Dai XZ, Shen C (2015) Identification method for low frequency oscillation strongly correlated generators based on power supply on the port. Autom Electr Power Syst 39(16):65-70. doi:10. 7500/AEPS20141015003

[23] Huang NE, Shen SS (2005) Hilbert-Huang transform and its applications. World Scientific Press, Singapore

[24] Peng JCH, Kirtley JL (2014) An improved empirical mode decomposition method for monitoring electromechanical oscillations. In: Proceedings of the 2014 IEEE PES innovative smart grid technologies conference (ISGT'14), Washington, 19-22 Feb 2014, 5 pp

[25] Zarraga FL, Rios AL, Esquivel P et al (2009) A Hilbert-Huang based approach for on-line extraction of modal behavior from
PMU data. In: Proceedings of the 2009 North American power symposium (NAPS'09), Starkville, 4-6 Oct 2009, 6 pp

[26] Yang DC, Rehtanz C, Li Y et al (2013) A hybrid method and its applications to analyse the low frequency oscillations in the interconnected power system. IET Gener Trans Distrib 7(8):874-884

[27] Amoud H, Snoussi H, Hewson D et al (2007) Intrinsic mode entropy for nonlinear discriminant analysis. IEEE Signal Process Lett 14(5):297-300

[28] Rodriguez R, Bila J, Mexicano A, et al. (2014) Hilbert-Huang transform and neural networks for electrocardiogram modeling and prediction. In: Proceedings of the 10th international conference on natural computation (ICNC'14), Xiamen, 19-21 Aug 2014, pp 561-567

Chen SHEN received the B.Eng., M.Eng., and Ph.D degrees from Tsinghua University, Beijing, China, in 1993, 1995, and 1998, respectively, all in electrical engineering. From 1998 to 2001, he worked as a Postdoctoral Research Fellow for the Electrical Engineering and Computer Science Department at the University of Missouri-Rolla. In 2001, he joined ISO New England as a Senior Engineer. From 2002 to 2009, he was an Associate Professor with the Department of Electrical Engineering, Tsinghua University. Since 2009, he has been a Professor with the Department of Electrical Engineering, Tsinghua University. His areas of research include power system analysis and control, distributed computing in power systems, and application of power electronic technology in power systems.

Zhi AN received the B.Eng. degree from Tsinghua University and M.Eng. degree from China Electric Power Research Institute, Beijing, China, in 2011 and 2014, respectively, all in electrical engineering. From 2014 to 2016, he was a Ph.D student with the Department of Electrical Engineering, Tsinghua University and later became a Ph.D candidate. His area of research is power system analysis and control.

Xianzhong DAI received the B.Eng. degree from Zhengzhou University and M.Eng. Ph.D degrees from Tsinghua University, in 2008, 2011 and 2015, respectively, all in electrical engineering. His area of research is power system analysis and control.

Wei WEI received the B.Eng. and Ph.D degrees from Sichuan University, Chengdu, China, in 2007 and 2012, respectively, all in electrical engineering. Since 2012, he has been an engineer in Electric Power Research Institute of Sichuan Electric Power Company. He has also been a Postdoctoral Research Fellow for the Department of Electrical Engineering, Tsinghua University since 2014. His area of research is power system analysis and control.

Lijie DING received the B.Eng. and Ph.D degrees from Zhejiang University, Hangzhou, China, in 2003 and 2008, respectively, all in electrical engineering. Since 2008, he has been an engineer in Electric Power Research Institute of Sichuan Electric Power Company. His area of research is power system analysis and control. 